\title{
3-D IMAGING, ANALYSIS AND MODELLING OF POROUS CEREAL PRODUCTS USING X-RAY MICROTOMOGRAPHY
}

\author{
GERARD VAN DALEN ${ }^{1}$, PETER NOOTENBOOM ${ }^{1}$, LUCAS J VAN VLIET ${ }^{2}$, LENNARD VOORTMAN ${ }^{2}$ \\ AND ERIK ESVELD ${ }^{3}$ \\ ${ }^{1}$ Advanced Measurement and Imaging, Unilever R\&D, Vlaardingen, The Netherlands; ${ }^{2}$ Quantitative Imaging \\ Group, Department of Imaging Science and Technology, University of Technology, Delft, The Netherlands; \\ ${ }^{3}$ Agrotechnology \& Food Sciences Group, AFSG Food Quality, University and Research Centre, Wageningen, \\ The Netherlands \\ e-mail: Gerard-van.Dalen@unilever.com; Peter.Nootenbom@unilever.com; lvvliet@mail.tn.tudelft.nl; \\ voortman@gmail.com; Erik.Esveld@wur.nl \\ (Accepted November 3, 2007)
}

\begin{abstract}
Efficient design of multi-component food products containing dry and wet components such as biscuits with a moist fruit filling, is of growing interests for food industry. Technology is needed to prevent or reduce water migration from the moist filling to the dry porous cereal material. This can be done by using moisture barrier systems. Knowledge of the microstructure and its relation to water mobility is necessary to develop stable products. This paper describes a study that uses X-ray microtomography $(\mu \mathrm{CT})$ for the characterisation and visualisation of the 3-D structure of crackers with different porosity, coated biscuit shells and soup inclusions. $\mu \mathrm{CT}$ was used for imaging the inner cellular structure of the cereal matrix or to analyse the integrity of moisture barriers applied on the cereal product. 3-D image analysis methods were developed to obtain quantitative information about the cellular matrix which can be used as input for simulation models for moisture migration. The developed 3-D image analysis method maps the open cellular structure onto a network (graph) representation in which the nodes correspond to the pores and the vertices to the pore-topore interconnection. The pores (nodes) have properties such as volume, surface area and location whereas the vertices have properties such as direct (open connection) and indirect (separated by a single lamella) area. To check the segmentation and network description a model for pore to pore resistance was used. The obtained results demonstrate the potential of $\mu \mathrm{CT}$ and 3-D image analysis for extracting structural information which can be used in models for the moisture penetration in a cellular bakery product.
\end{abstract}

Keywords: cellular food products, hydration, microstructure, X-ray microtomography ( $\mu C T)$, XRT, XMT.

\section{INTRODUCTION}

Today's western societies demand food products with superior taste, texture, freshness and shelf life that are ready-to-eat or quick and easy to prepare. A modern food industry continuously innovates to develop these high quality food products. An important segment of food innovation comprises cereal products such as bread, snacks and (preprocessed) staple foods. Crispiness and crunchiness are important features in term of consumer appreciation of dry porous cereal materials. In general, the crispy/crunchy perception of these materials decreases in storage due to a change in water content (uptake from surrounding media), change in water distribution or change in mobility of water. The rate of this decrease depends on the product composition, the microstructure and the storage condition. Efficient design of multi-component food products containing dry and wet components such as biscuits with a moist fruit filling, is of growing interests for food industry. Technology is needed to prevent or reduce water migration from the moist filling to the dry porous cereal material. This can be done by using moisture barrier systems. Knowledge of the microstructure and its relation to water mobility is necessary to develop stable products. Magnetic Resonance Imaging (MRI) allows the real time visualisation of the effect of hydration in terms of transport/diffusion rate and spatial distribution of structural changes (Mohorič et al., 2004; Ramos-Cabrer et al., 2005; van Dalen et al., 2005). X-ray microtomography ( $\mu \mathrm{CT}$ ) on the other hand allows for high resolution 3-D visualisation and characterisation (e.g. porosity assessment) of the non-hydrated material before and after certain durations of hydration. $\mu \mathrm{CT}$ has proven to be a very useful technique for the non-invasive 
visualisation and measurement of the internal microstructure of cellular food products, such as porous rice kernels and whipped cream (van Dalen et al., 2003), aerated chocolate and muffins (Lim et al., 2004), bread (Falcone et al., 2004; 2005; Lassoued et al., 2007), cornflakes (Chaunier et al., 2007), dough (Mousavi et al., 2005), extruded starches (Babin et al., 2007), French fries (Miri et al., 2006) and biopolymer foams (Trater et al., 2005).

In order to understand the relation between microstructure and water uptake mathematical simulation models will be developed. For these models a number of free parameters are needed e.g. initial and final moisture content, structural parameters and type of diffusion. Structural parameters can be derived from the 3-D structure visualised by $\mu \mathrm{CT}$. The final models have to be validated using MRI. Modelling and MRI imaging will be reported in a separate paper. This paper describes a study that uses $\mu \mathrm{CT}$ to image and visualise the internal structure of dry crackers with different porosity, coated biscuits shells and soup inclusions. 3-D image analysis methods were developed to obtain quantitative information about the cellular matrix which can be used as input for simulation models. Information about the properties of each pore, such as volume, surface area, location and properties of connections between pores such as direct (open connection) and indirect (separated by a single lamella) areas were analysed. In this case larger volumes can be modelled than by finite element analysis using the 3 -D volumetric mesh of the porous structure (Babin et al., 2005).

\section{MATERIAL AND METHODS}

Samples: The cereal based samples were produced locally. Differences in microstructure were achieved by application of different manufacturing processes to the same dough formulation. Wheat flour based soup inclusions $(0.5-1 \mathrm{~cm}$ in diameter) were coated with $10-16 \%$ vegetable fat. Biscuit shells of $3.5 \times 3.5$ $\times 2.0 \mathrm{~cm}$ were coated internally with a layer of lipid

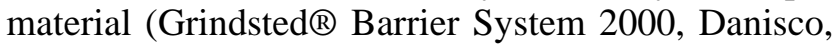
Denmark), which is a blend of wax and acetylated monoglyceride. A flour/fat/sugar ratio of 100/34/15 was used to make a dough that was suitable for lamination. Series of carbohydrate based crackers of different porosity were equilibrated at constant hydration levels, Aw of $\sim 0.15$ ). The cracker dough was made of $64 \%$ flour, $25 \%$ water, $0.12 \%$ salt, $0.12 \%$ yeast, $6.4 \%$ sunflower oil, $0.12 \%$ dextrose monohydrate and $0.003 \%$ Bakezyme B500. Crackers were produced using different proofing times (ranging from 10 to $130 \mathrm{~min}$ ). For imaging, crackers of $8 \times 8 \times 1 \mathrm{~cm}$ were cut into sub-samples of about $6 \times 6 \times 6 \mathrm{~mm}$.

$\mu C T$ : Samples were imaged using a Skyscan 1072 desktop $\mu$ CT system (Belgium, http:// www.skyscan.be, Sasov, 1987). Power setting of $50 \mathrm{kV}$ and $100 \mu \mathrm{A}$ were used. A low voltage of $50 \mathrm{kV}$ results in a much better stability than the maximum voltage of $100 \mathrm{kV}$ and gives a better contrast for samples with a low absorbing matrix as cereals. The transmission of the conical X-ray beam through the sample is recorded by a CCD camera with $1024 \times 1024$ pixels. $\mu$ CT can probe the 3-D microstructure of samples non-invasively up to a few millimetres across with an axial and lateral resolution down to about $6 \mu \mathrm{m}$. The contrast in $\mu \mathrm{CT}$ images is based on the difference in absorption of Xrays by the constituents of the sample (e.g. fat, carbohydrate and air). In cereal materials the contrast is mainly caused by a difference in density. $\mu \mathrm{CT}$ produces two-dimensional images of projections of the sample. A set of flat cross sections was obtained after tomographical reconstruction of images acquired under different rotations over 180 degrees with a step size of 0.45 degrees. The acquisition time for one projection was $2.8 \mathrm{~s}$ resulting in a total acquisition and read-out time of about $40 \mathrm{~min}$. Samples are not influenced by the high energy X-rays allowing long acquisition times. Crackers and coated biscuits were fixed on a specimen holder using glue. Soup inclusions were imaged using plastic cylindrical sample holder with an inner diameter of $11.2 \mathrm{~mm}$.

Image analysis and visualisation: For image processing and analysis the image analysis toolbox (DIPlib vers. 1.5.0 from the Delft University of Technology, NL, http://www.ph.tn.tudelft.nl/DIPlib/) running under MATlab (version 7.1, R14, SP3 from MathWorks) was used. For visualisation in 3-D space, isosurface rendering was used (Amira 4.1 from Mercury Computer Systems). The software was used on an IBM IntelliStation Z Pro Workstation running under Windows XP.

\section{DIFFUSION MODELLING}

To model the moisture transport in the structure of the porous cracker material a 3-D network representation will be constructed of the actual sample. The features will be extracted from the 3-D $\mu \mathrm{CT}$ voxel space by means of image analysis techniques. 


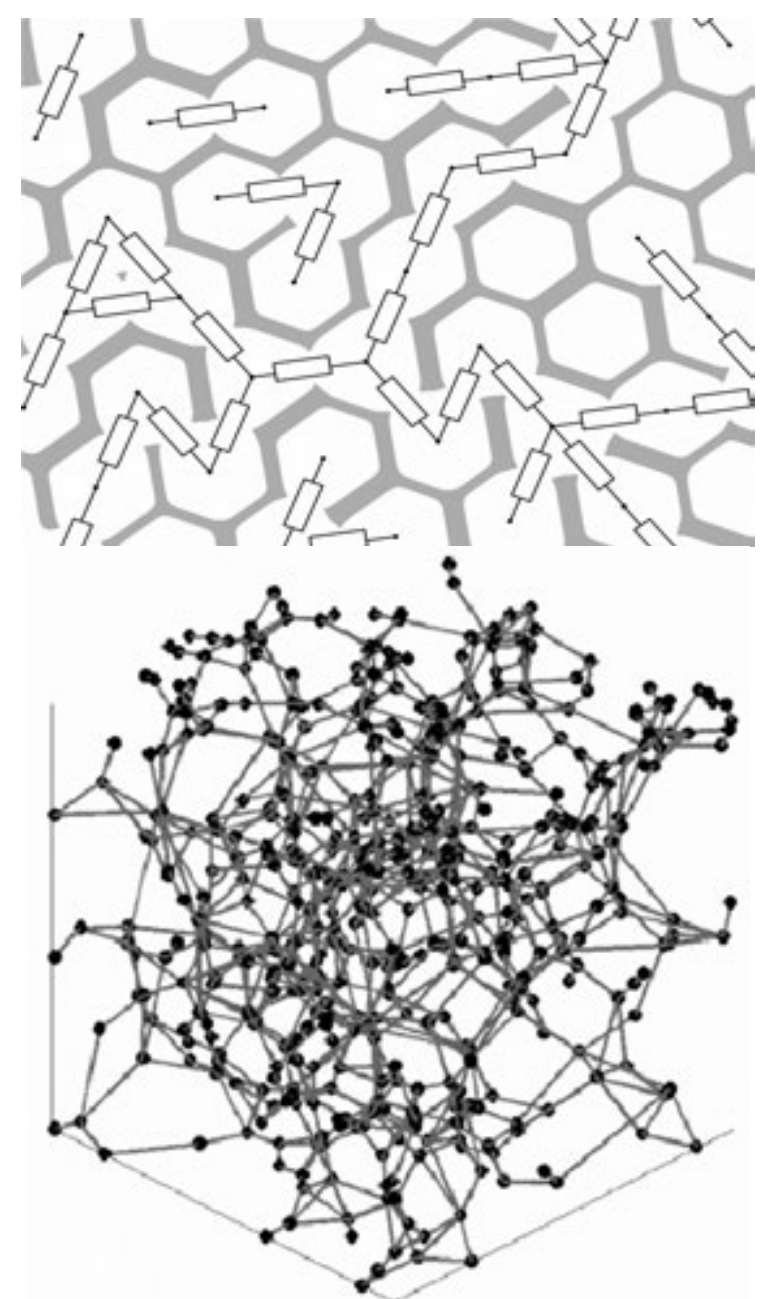

Fig. 1. Schematic 2-D and 3-D representation of a network showing the vapour conductance path through an open cellular matrix.

A short description of the diffusion modelling will be given and which features have to be analysed (development of diffusion models will be handled in a separate paper). For modelling only the macroscopic moisture transport by diffusion through the vapour phase and the local diffusive penetration in the solid will be taken into account. The product is not supposed to be in direct contact with liquid water. The solid matrix will swell by a different mechanism when in contact with liquid water, as opposed to purely Fickian diffusion (due to a concentration gradient) considered here. Capillary and surface forces, which drive the transport of liquid water trough a matrix, will not be included in the model. $\mu \mathrm{CT}$ imaging will show that nearly all voids are connected in 3-D space (see results) resulting in a continuous gas phase. Every cell has a free gas diffusion path to a neighbour. Given the fact that the moisture conductivity trough the gas phase is initially several orders of magnitude higher than the conductivity trough the condensed phase, any cell-tocell moisture flux trough the condensed phase will be disregarded. Furthermore, it is assumed that the moisture penetration would be symmetrical from both sides. Fig. 1 shows a schematic representation of a connected network structure. The individual cells are the nodes of the network and are connected with at least one vapour conductance path to a neighbour. The surface areas of these connection points together with the cell diameters are sufficient to characterise the cell-to-cell vapour conductance. Each node will also contain a term to model the moisture sorption in the lamellae. As input the lamellae thickness of each cell over their surrounding wall surface area is needed.

\section{IMAGE ANALYSIS OF A CONNECTED CELLULAR MATRIX}

To construct a 3-D network model, the connected void space has to be segmented into individual pores, which are represented by nodes in the network (Fig. 1). These nodes all have different properties, such as volume, surface area and location. The vertices describe the relation between two nodes. The network properties will be stored in several matrices. First a cubic part of the original 3-D $\mu \mathrm{CT}$ image of a cracker sample was selected by cropping the stack of 2-D $\mu C T$ images, excluding the sample edges which may have been affected by physical cutting of the sample from the total cracker. The random noise of the $\mu \mathrm{CT}$ images was reduced using a 3-D median filter. For segmentation of the voids from the solid matrix manual thresholding was used, resulting in a 3-D binary image of the voids. Isolated voids smaller than 40 voxels were removed. The connected voids were separated using a watershed transform of the Euclidean distance map of the solid material (Fig. 2). 


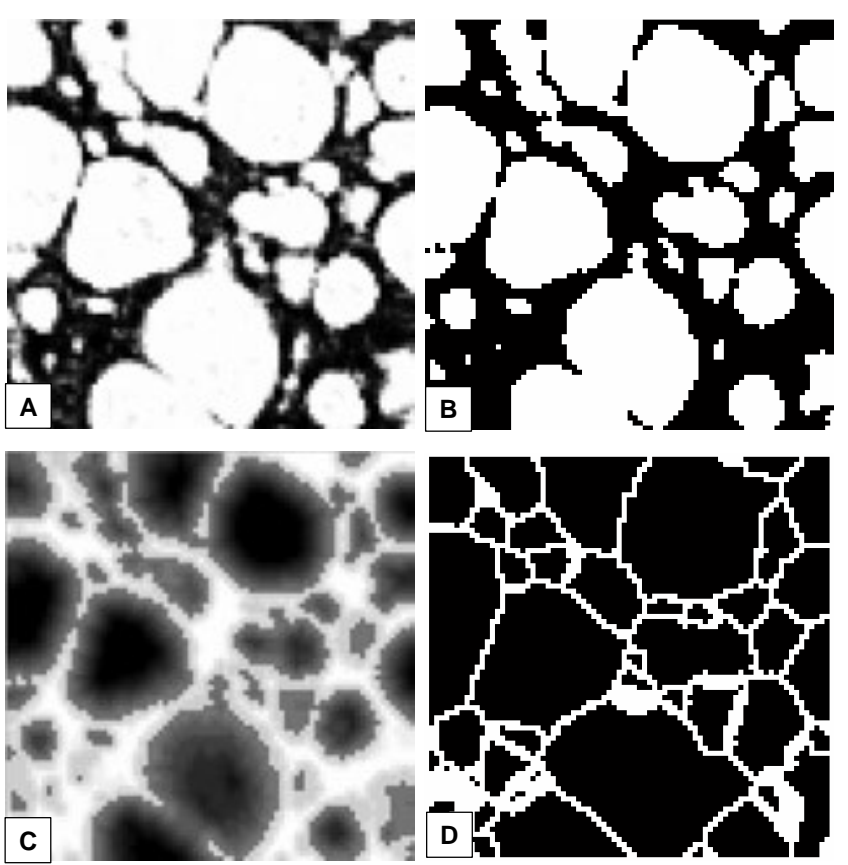

Fig. 2. Segmentation of the void space showing a 2-D section $(81 p x \times 81 p x)$ of the original grey scale $\mu C T$ image of a cracker $(A)$, the binary image after removal of small isolated voids $(B)$, the result of the distance transform (dt) of $B(C=d t(\sim p)-d t(p)-p)$ and the result of the watershed transform on image $C(D)$.

The watershed transform was used with an 18connected neighbourhood and regions less deep than 0.38 were merged to prevent over-segmentation. The boundaries generated by the watershed transform are used to label each pore and its surrounding solid material (Fig. 3).

The labelling process generates different labels for the pores which are used as index in the corresponding matrix. Mapping was done by measuring both labelled images. The volume, surface area and location of the pores were measured using the labelled pore image. To construct the network model the boundaries between two adjacent pores have to be known. When the edges of two pore regions overlap there is a boundary. The edges of each region were calculated and saved as separate images. This was done by growing each individual region selected from the region labels by using a binary dilation step and multiplying the results with the boundaries obtained by the watershed method. The shared edges were calculated by multiplying the boundaries of each region (Fig. 4). The areas of the direct - and indirect pore connections (Ad and $\mathrm{Ai}$ ) were calculated from the shared edges within the void space and the shared edges within the matrix. The developed method describes a sample (with a cellular open structure) by a collection of matrices. A diagonal matrix stores the pore properties, whereas the offdiagonal elements denote the pore-to-pore relations.
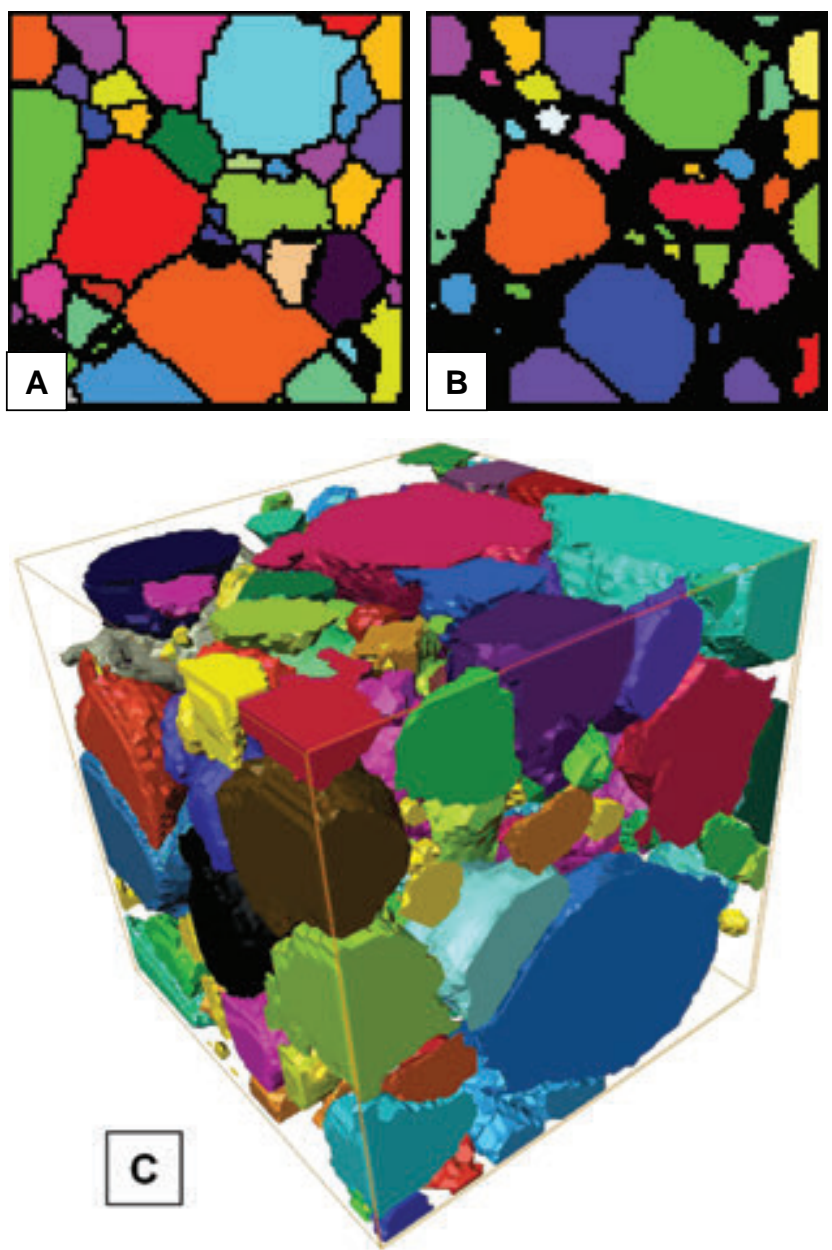

Fig. 3. 2-D sections of labelled regions surrounding the pores (A) and labelled pores (B) with 3-D labelled image of pores (C).

The single pore properties describe the pore volume, surface area etc. The pore diameters were calculated from the volume of the individual pores. The so called equivalent pore diameter is the diameter of a sphere having the same volume as the pore. The volume is the number of pixels within the pore, which is straightforwardly determined by counting.

The pore-to-pore properties describe relations between adjacent pores. The network can be used to calculate the pore-to-pore resistance. To check the segmentation and network description a very crude model for point to point resistance was used. Development of diffusion models is outside the scope of this paper. 


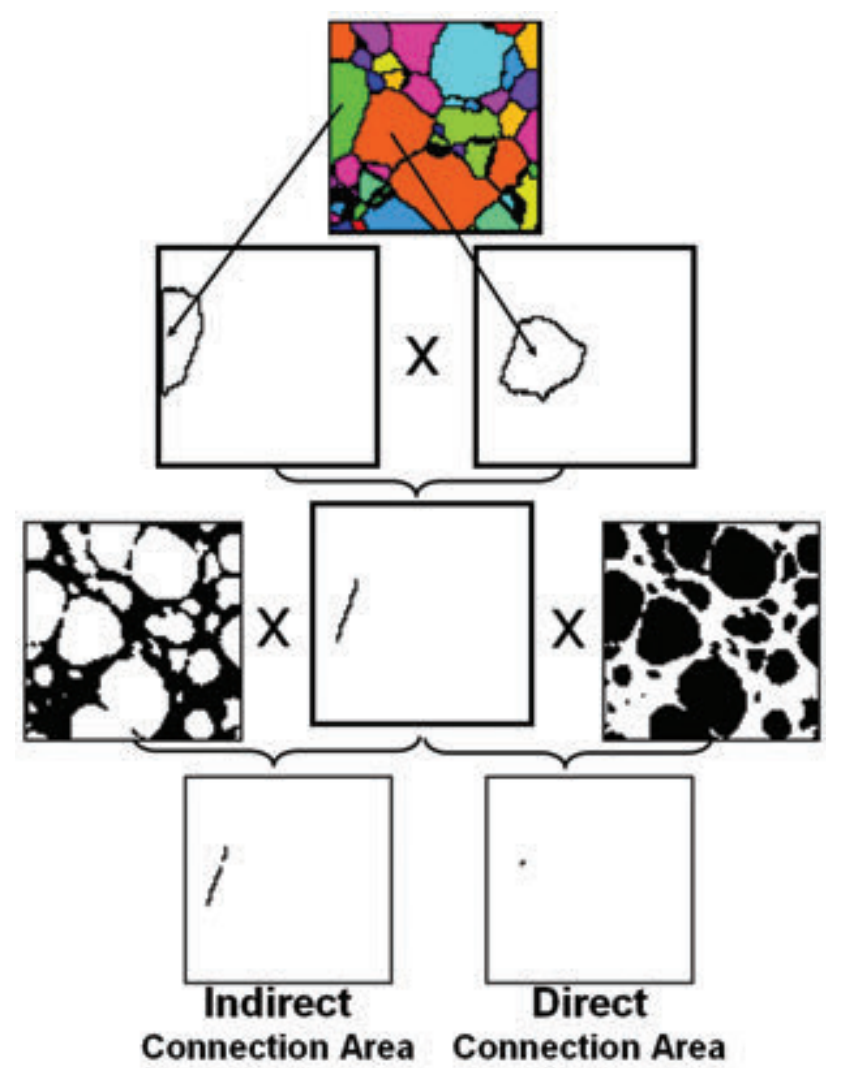

Fig. 4. Identification of the direct - and indirect pore connections.

As approximation of the conduction between two pores ( $i$ and $j$ ) the following formula was used:

$$
C_{i j}=\left(A d_{i j}+\frac{A i_{i j}}{100}\right) *\left(1 / S A_{-} \text {pore }\right),
$$

in which the area between direct connected pores $(A d)$, the area of indirect connected pores $(A i)$ and the pore surface (SA_pore) are used (1/SA_pore is a diagonal matrix with the pore surfaces on its diagonal). The point-to-point resistance $\left(\mathrm{R}_{\mathrm{i}, \mathrm{j}}\right)$ was calculated from the conduction using the resistance theorem described by $\mathrm{Wu}$ (2004) by using the eigenvalues and eigenfunctions of the Laplacian matrix associated with the network. First the Lagragian of the network was created:

$$
L=\left(\begin{array}{cccc}
C_{1} & -C_{12} & \cdots & -C_{1 n} \\
-C_{21} & C_{2} & \cdots & -C_{2 n} \\
\vdots & \vdots & \ddots & \vdots \\
-C_{n 1} & -C_{n 2} & \cdots & C_{n}
\end{array}\right)
$$

where $C_{i}=\sum_{j=1}^{n} C_{i j}$.

When the Laplacian has nonzero eigenvalues $l$ with corresponding eigenvectors

$$
\Psi_{k}=\left(\Psi_{k 1}, \Psi_{k 2}, \ldots, \Psi_{k n}\right), k=2,3, \ldots, n,
$$

then the resistance between two nodes $i$ and $j$ is given by

$$
R_{i j}=\sum_{k=2}^{n} \frac{1}{l_{k}}\left|\Psi_{k i-} \Psi_{k j}\right|^{2} .
$$

\section{RESULTS}

\section{COATED CEREALS}

Fig. 5 shows a $\mu \mathrm{CT}$ image of a lipid coated biscuit shell. The lipid barrier layer is clearly visible as a homogeneous grey region. Only a part of the cup shaped shell was imaged due to a limited field of view (image width of $18 \mathrm{~mm}$ at a pixel size of 18 $\mu \mathrm{m})$. For this purpose the edge was cut out of the cup. This is the most difficult part to cover in the coating process. The images can be used for the determination of the layer thickness and for the detection of small holes penetrating the barrier layer, as seen for example at the corner of the biscuit in the $\mu \mathrm{CT}$ image of the horizontal cross section shown in Fig. 5A. Small holes in the barrier layer of filled shells will cause product failure after a couple of days showing swelling of the shell material, whereas thin areas in the barrier layer result in slow softening of the shell material (water permeability depends on the thickness of the barrier layer). 


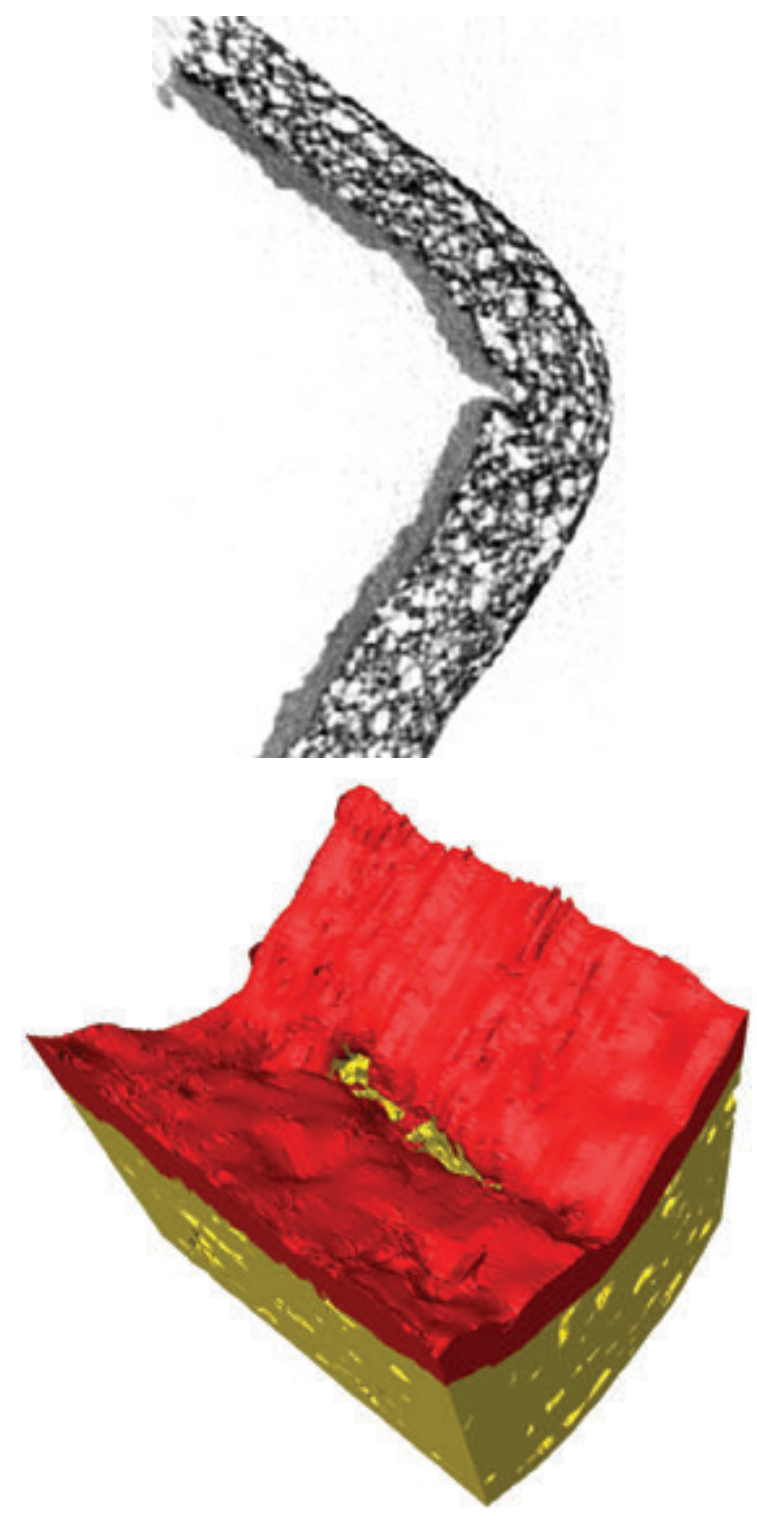

Fig. 5. $\mu C T$ image of a horizontal cross section (top) of a coated cereal shell with 3-D reconstruction (bottom) of the barrier layer (red) and cereal shell (yellow), thickness $=2.3 \mathrm{~mm}$ (shell) and $0.5 \mathrm{~mm}$ (coating), cubic voxels of $18 \mu \mathrm{m}$.

Soup inclusions with three different types of structure were produced, based on 2-, 4- and 8-layers dough. The spherical inclusions were coated with a layer of fat. 3-D $\mu \mathrm{CT}$ images with resolution of $15 \mu \mathrm{m}$ were obtained, showing differences in internal structure of the samples (Fig. 6). In the soup inclusions based on 2- and 4 layers relatively big internal air voids were found surrounded by layered material. While, in the 8 layered type of soup inclusion smaller pores were distributed more randomly through the sample. The integrity of the fat coating was assessed by means of differences in X-ray attenuation between cereal matrix and fat coating. In most cases this revealed non-uniform coating of the product surface. Correlating $\mu \mathrm{CT}$ with
MRI hydration experiments showed that regions of the sample surface which were poorly coated were responsible for faster initial hydration (Weglarz et al., 2007).

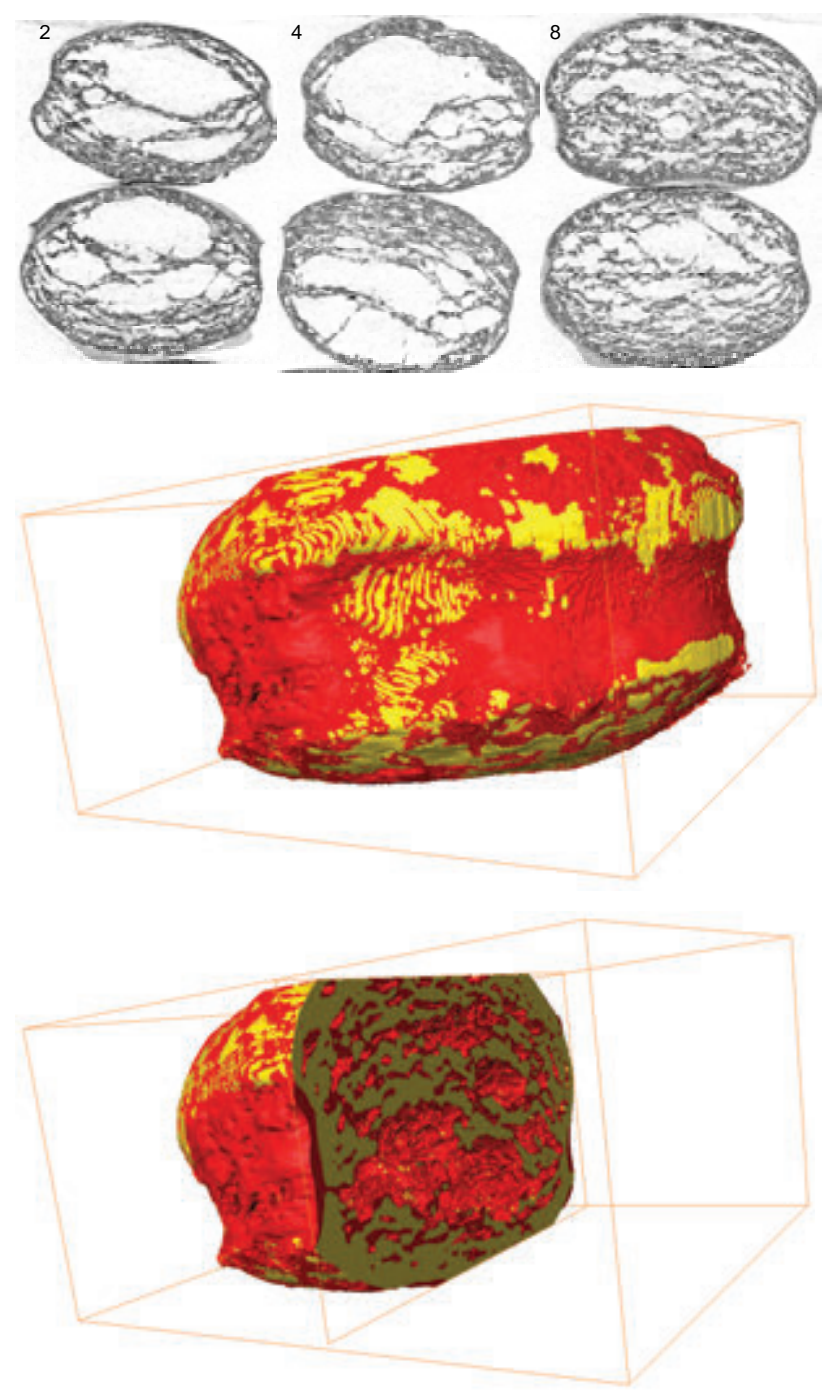

Fig. 6. 2-D cross-sections through 3-D $\mu C T$ images of soup inclusions based on 2-, 4- and 8-layer structures (top from left to right); 3-D $\mu C T$ visualisation of nonuniform distribution of fat coating (red colour), box size $=12.0 * 6.0 * 6.0 \mathrm{~mm}$.

\section{CELLULAR CEREAL MATRIX}

Fig. 7 shows the $\mu \mathrm{CT}$ images of 5 different crackers used to study the moisture penetration in cellular cereal materials without moisture barriers. The crackers were produced using proofing times of $10,25,60$, 115 and $130 \mathrm{~min}$. respectively for samples A-E. All samples were imaged at a low magnification factor $(1 \mathrm{px}=15.2 \mu \mathrm{m})$ showing the whole cellular structure including the top and bottom crust. A sample of the central part of cracker A (without crust) was imaged at a higher magnification $(1 \mathrm{px}=7.8 \mu \mathrm{m})$. 


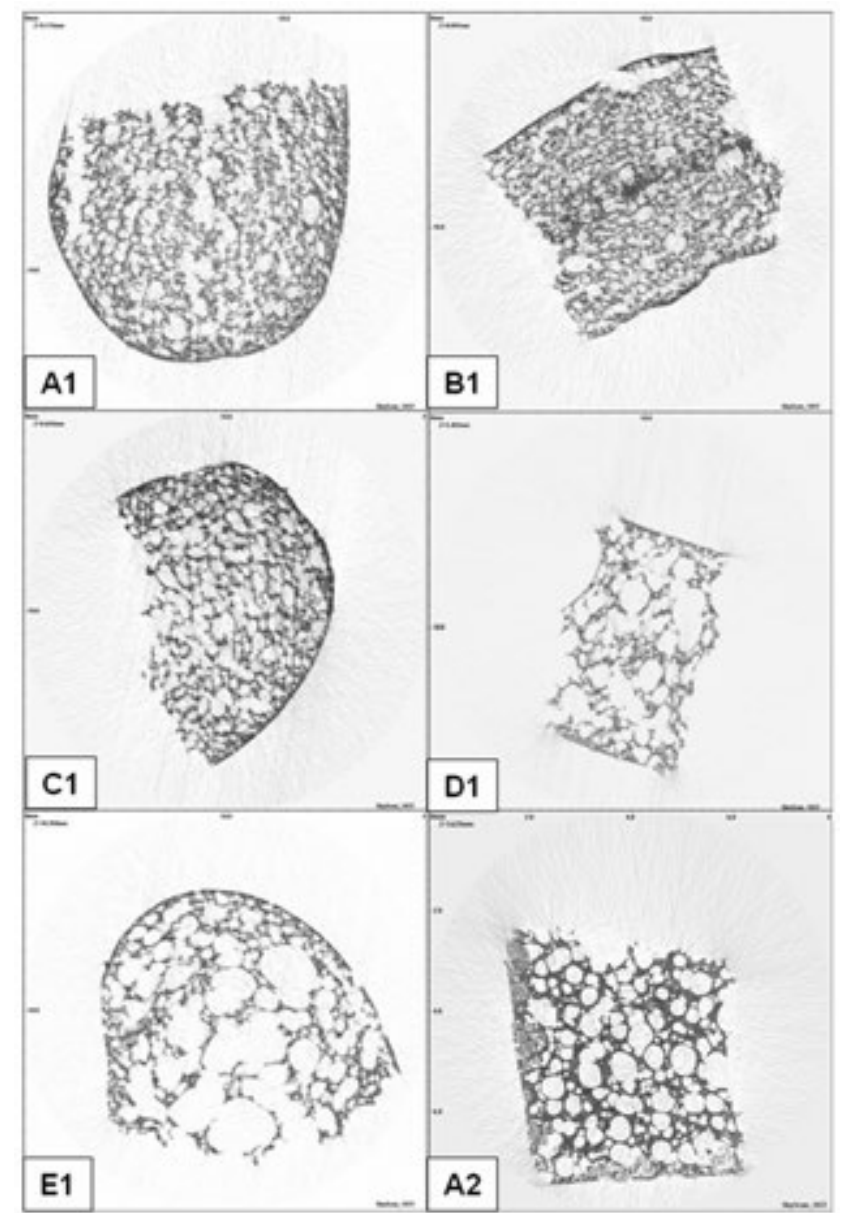

Fig. 7. 2-D horizontal cross-sections through 3-D $\mu C T$ images of cracker samples produced using proofing times of $10 \mathrm{~min}(\mathrm{~A}), 25 \mathrm{~min}(B), 60 \mathrm{~min}(\mathrm{C}), 115 \mathrm{~min}$ (D) and $130 \mathrm{~min}(E)$. Image width $=15.6 \mathrm{~mm}$ for A1E1 and $8.0 \mathrm{~mm}$ for $\mathrm{A} 2$.

The dense layer on the edge of image A2 in Fig. 7 is a cutting artefact and will be removed for further analysis. An increase in proofing time resulted in a rather step-wise increase in pore size and porosity. Proofing times ranging from 10 to 60 min (sample AC) resulted in a low porosity and small pore sizes and proofing times of 115 and $130 \mathrm{~min}$ (sample D-E) resulted in a high porosity and large pore sizes. The porosity of samples A-C, expressed as the mean value of the total area fraction of pores in each cross section of the total imaged volume ranges from 45 to $54 \%$ and the porosity of samples D-E ranges from 72 to $78 \%$. The volume weighted average pore diameter ranges from 0.4 to $0.6 \mathrm{~mm}$ for sample A-C and from 1.2 to $1.3 \mathrm{~mm}$ for sample D-E. The diameter is expressed as the equivalent sphere diameter which is the diameter of a sphere having the same volume as the pore. The volume is the number of pixels within the pore, which is straightforwardly determined by counting. Image analysis of $\mu \mathrm{CT}$ images of these crackers showed that nearly all the void space (95\%) is connected in 3-D space (at a pixel size of $7.8 \mu \mathrm{m}$ ). $\mu \mathrm{CT}$ images of sample A with a pixel size of $7.8 \mu \mathrm{m}$ and images of sample D with a pixel size of $15.2 \mu \mathrm{m}$ were selected to develop a method to analyse the single pore, and pore-to-pore properties of a fully connected cellular matrix, constructing a network representation that can be used as input for diffusion modelling. The number of connections of each pore of cracker sample A and $\mathrm{D}$ as function of the pore size are shown in Fig. 9. The number of connections increases with increasing pore size. In sample $\mathrm{D}$, containing large pores, the number of direct connections is on average much higher than for sample A containing small pores. These observations are in agreement with the expectations that large pores will generally have more connections.

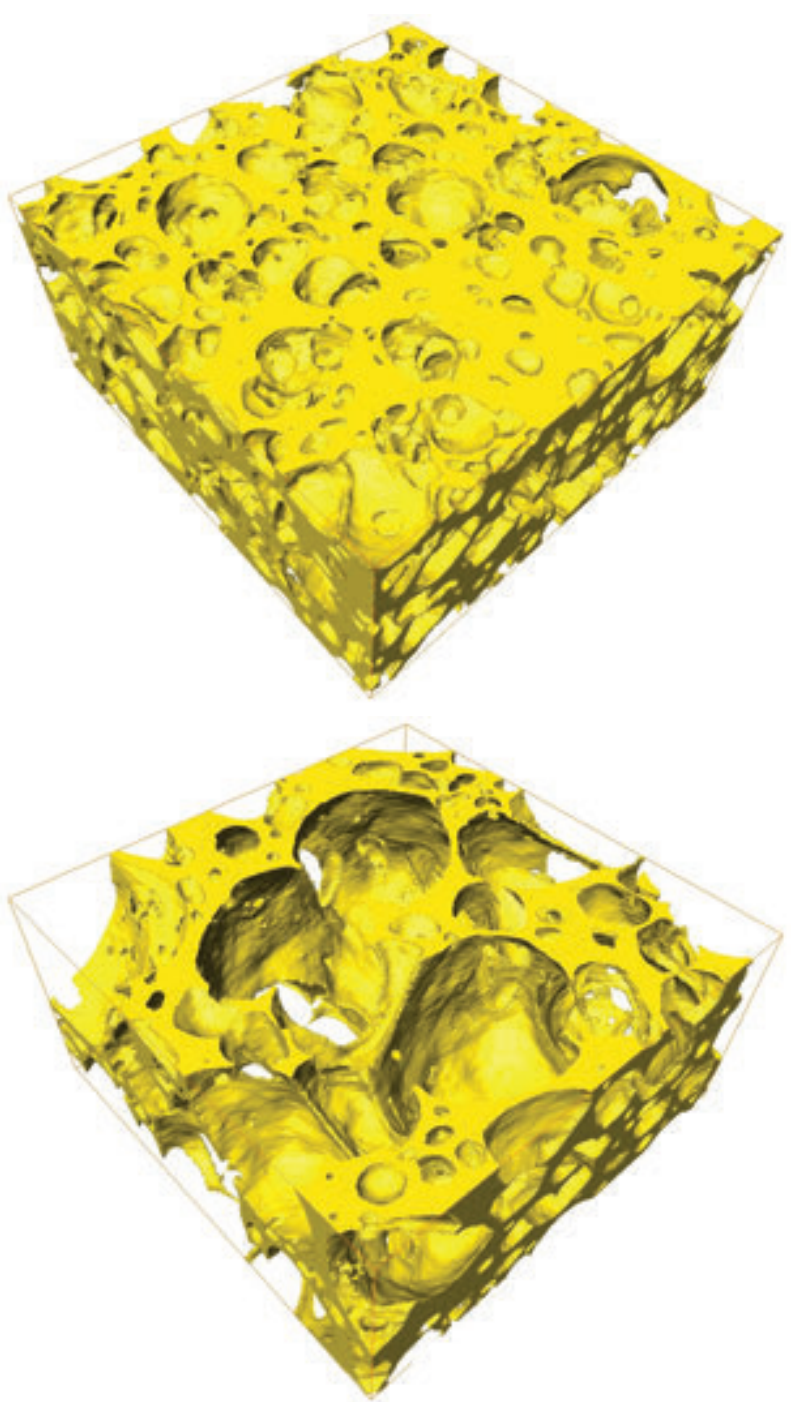

Fig. 8. 3-D visualisation of $\mu C T$ images of cracker sample $A$ (top) and $D$ (bottom), produced using proofing times of $10 \mathrm{~min}$ and $115 \mathrm{~min}$ (box size = $3.3 \times 3.2 \times 1.6 \mathrm{~mm}^{3}$ ). 
The relation between the pore-to-pore resistance $\left(\mathrm{R}_{\mathrm{ij}}\right)$ and the pore-to-pore distance is shown in Fig. 10 for cracker sample A and D. As can be expected, pores that lay further apart have on average a larger resistance as two adjacent pores. A linear relation was observed between the resistance and distance. The average pore-to-pore resistance of sample $\mathrm{D}$, containing large pores is much lower than for sample A, containing small pores. The slope of the linear relation between the resistance and distance is about a factor 4 higher for sample A than for sample D.
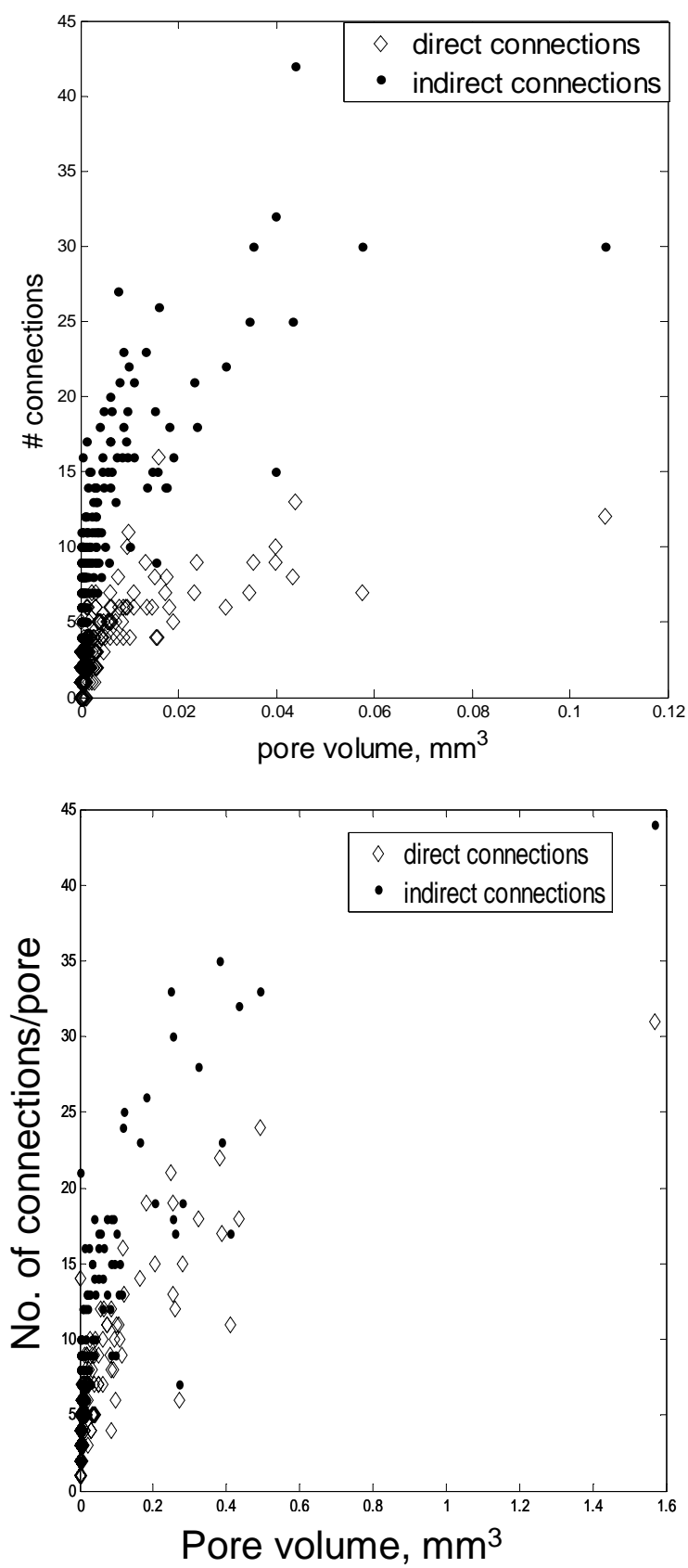

Fig. 9. The number of direct - and indirect (lamellae) connections of each pore as function of the pore size for cracker sample A (top) and D (bottom).
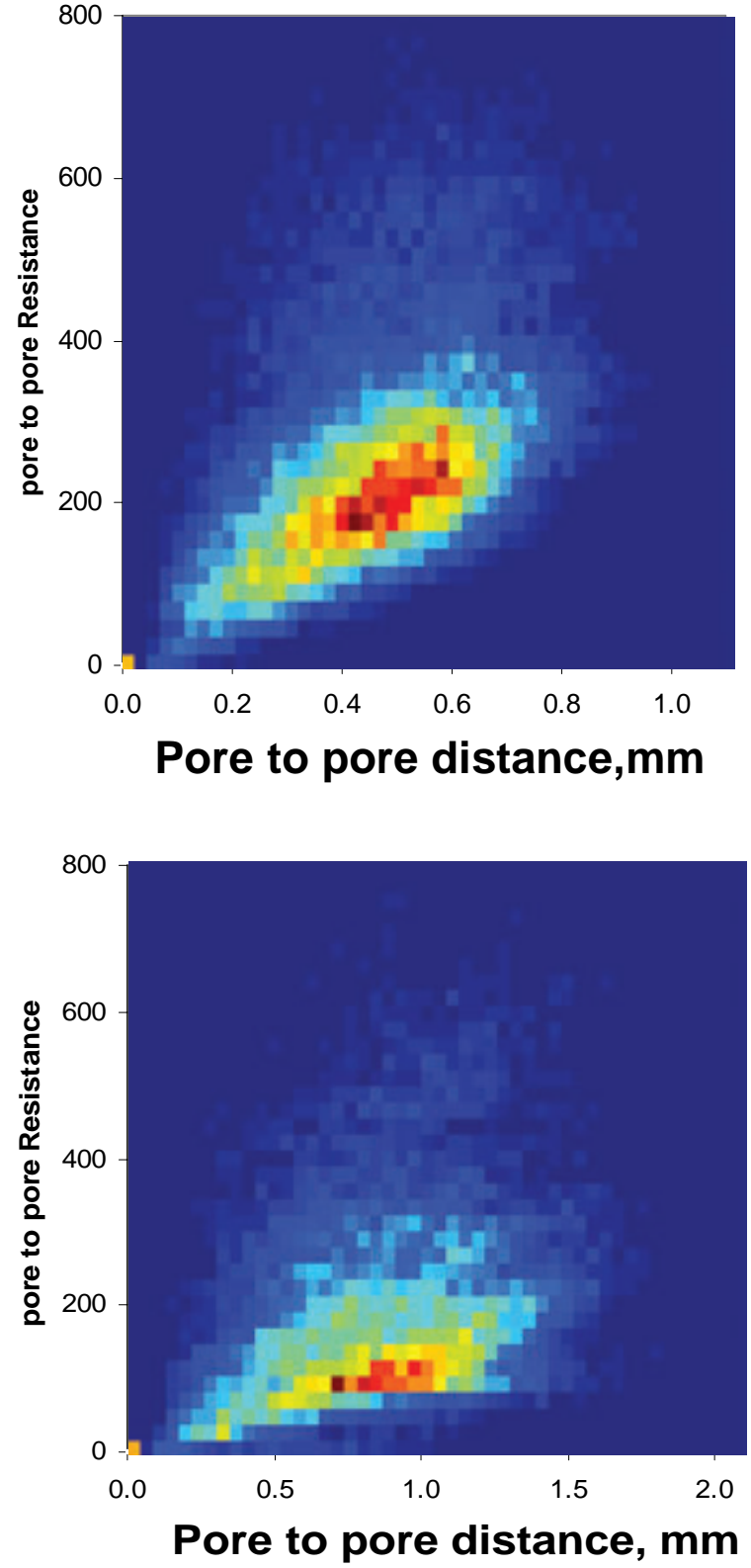

Fig. 10. Relation between the pore to pore resistance and the distance between each pore centre of cracker sample A (top) and D (bottom) visualised as a two dimensional histogram.

\section{CONCLUSION}

$\mu C T$ proved to be a very useful technique for the non-invasive 3-D visualisation and quantitative analysis of the microstructure of cellular cereal products. It can be used to study the inner cellular structure of the cereal matrix or to assess the integrity of moisture barriers applied on cereal product. The obtained quantitative information can be used as input for simulation models for moisture diffusion. The developed 3-D image analysis method describes the open cellular structure 
by a collection of matrices which account for its single pore properties (e.g. volume, surface area and location) and pore-to-pore properties (areas of the direct - and indirect pore connections).

\section{REFERENCES}

Babin P, Della Valle G, Dendievel R, Lassoued N, Salvo L (2005). Mechanical properties of bread crumbs from tomography based Finite Element simulations. J Mat Science 40:5867-73.

Babin P, Della Valle G, Dendievel R, Lourdin D, Salvo L (2007). X-ray tomography study of the cellular structure of extruded starches and its relations with expansion phenomenon and foam meachanical properties. Carbohydrate Pol 68:329-40.

Chaunier L, Della Valle G, Lourdin D (2007). Relationships between texture, mechanical properties and structure of cornflakes. Food Res Int 40:493-503

Falcone PM, Baiano A, Zanini F, Mancini L, Tromba G, Montanari F, et al. (2004). A novel approach to the study of bread porous structure: Phase-contrast $\mathrm{x}$-ray microtomography J Food Science 69(1):FEP38-43.

Falcone PM, Baiano A, Zanini F, Mancini L, Tromba G, Dreossi D, et al. (2005). Three-dimensional quantitative analysis of bread crumb by X-ray microtomohraphy. $\mathrm{J}$ Food Sciences 70(3):E265-72.

Lassoued N, Babin P, Della Valle G, Devaux MF, Réguerre AL (2007). Granulometry of bread crumb grain: Contributions of 2D and 3D image analysis at different scale. Food Research Int 40:1087-97.

Lim KS, Barigou M (2004). X-ray micro-computed tomography of cellular food products. Food Research Int 37(10):1001-12.

Miri T, Bakalis S, Bhima SD, Fryer PJ (2006). Use of
$\mathrm{X}$-ray Micro-CT to characterize structure phenomena during frying, http://iufost.edpsciences.org.

Mohorič A, Vergeldt F, Gerkema E, de Jager A, van Duynhoven J, van Dalen G, et al. (2004). Magnetic resonance imaging of single rice kernels during cooking. J Magn Res 171:157-62.

Mousavi R, Miri T, Cox PW, Fryer PJ (2005). A novel technique for ice crystal visualization in frozen solids using X-ray micro-computed tomography, J Food Sciences 70(7):E437-42.

Ramos-Cabrer P, van Duynhoven JPM, van Dalen G, Nicolay K (2005). MRI: assessment of water transport in food. Ciencia en la frontera 3(1):59-66.

Sasov A (1987). Microtomography. Part 1 and 2. J Microscopy 191(2):169-92.

Trater AM, Alavi S, Rizvi SSH (2005). Use of noninvasive X-ray microtomography for characterizing microstructure of extruded biopolymer foams. Food Res Int 38(6):709-19.

van Dalen G, Blonk JCG, van Aalst H, Luengo Hendriks CL (2003). 3-D imaging of foods using X-ray microtomography, GIT. Imaging \& Micr 5(3):18-21, http://clluengo.lbl.gov/Documents/GIT2003.pdf.

van Dalen G, van Duynhoven J, Blonk JCG, Mohorič A, Ramos-Cabrer P, van den Doel R (2005). Multi dimensional imaging of foods using magnetic resonance imaging, GIT. Imaging \& Micr 7(3):42-4.

Weglarz WP, Hemelaar M, van der Linden KA, Franciosi N, van Dalen G, Windt C, et al. (2008). Real-time mapping of moisture migration in cereal based food systems with Aw contrast by means of MRI. Food Chem 106:1366-74.

Wu FY (2004). Theory of resistor networks: the two-point resistance. J of Physics - A Mathematical and General 37(26):6653-73. 